\title{
Daratumumab: a first-in-class CD38 monoclonal antibody for the treatment of multiple myeloma
}

\author{
Larysa Sanchez $^{1 \dagger}$, Yucai Wang ${ }^{1 \dagger}$, David S. Siegel ${ }^{2}$ and Michael L. Wang ${ }^{3 *}$
}

\begin{abstract}
Daratumumab is a human monoclonal antibody that targets CD38, a cell surface protein that is overexpressed on multiple myeloma (MM) cells. Preclinical studies have shown that daratumumab induces MM cell death through several mechanisms, including complement-dependent cytotoxicity (CDC), antibody-dependent cell-mediated cytotoxicity (ADCC), antibody-dependent cellular phagocytosis (ADCP), and apoptosis. Given the encouraging efficacy and acceptable safety profile of daratumumab demonstrated in clinical trials, daratumumab has emerged as a novel treatment option for myeloma and became the first monoclonal antibody approved by the FDA for the treatment of MM.
\end{abstract}

\section{Background}

Advances in the treatment of multiple myeloma (MM), particularly the development of immunomodulatory drugs (IMiDs) and proteasome inhibitors (PIs) and the use of autologous hematopoietic stem cell transplantation, have led to significant improvement in overall survival in patients with MM $[1,2]$. Nevertheless, MM remains incurable and outcomes in the relapsed/refractory setting are very poor [3]. This underscores an urgent need for novel agents in the treatment of MM, especially in patients who have become refractory to currently available therapies [4]. In recent years, the introduction of monoclonal antibodies (mAbs) in MM therapy, notably mAbs targeting CD38 and SLAMF7, has been a promising step forward in improving treatment outcomes [5]. Here, we provide a brief overview of $\mathrm{CD} 38$ as a therapeutic target in MM and review available preclinical and clinical data on daratumumab, the first-in-class human anti-CD38 mAb approved for the treatment of MM.

\footnotetext{
*Correspondence: miwang@mdanderson.org

${ }^{\dagger}$ Equal contributors

${ }^{3}$ Department of Lymphoma/Myeloma, Division of Cancer Medicine,

University of Texas M. D. Anderson Cancer Center, 1515 Holcombe Blvd., Unit 429, Houston, TX 77030, USA

Full list of author information is available at the end of the article
}

\section{Targeting CD38 in multiple myeloma}

CD38 is a $46-\mathrm{kDa}$ type II transmembrane glycoprotein that is expressed on lymphoid and myeloid cells and also on non-hematopoietic tissues [6, 7]. Notably, CD38 is highly expressed on MM cells [8]. CD38 has been found to have multiple functions, including ectoenzymatic activity as well as receptor-mediated regulation of cell adhesion and signal transduction [7, 9]. The enzymatic activity of CD38 involves the conversion of nicotinamide adenine dinucleotide $(\mathrm{NAD}+)$ and nicotinamide adenine dinucleotide phosphate (NADP+) to cyclic adenosine diphosphate ribosyl (cADPR), ADPR, and nicotinic acid adenine dinucleotide phosphate (NAADP), substrates necessary for regulation of intracellular calcium signaling [6]. In initial studies investigating the receptor function of CD38, it was found that CD38 mediates weak cell binding to endothelium and plays a role in lymphocyte migration, as well as exhibits functional associations with surface molecules of $\mathrm{T}, \mathrm{B}$, and natural killer (NK) cells $[10,11]$. The role of CD38 in cellular adhesion was further delineated with the identification of CD31 as a cell surface ligand for CD38 on endothelial cells [12]. Deaglio et al. found that CD38/CD31 interactions resulted in trans-membrane signaling characterized by calcium mobilization and cytokine secretion [12]. CD38 ligation resulting in activation of T lymphocytes was found to induce secretion of interleukin (IL)-6, 
granulocyte-macrophage colony-stimulating factor (GMCSF), interferon- $\gamma$ (IFN- $\gamma$ ), and IL-10 cytokines [13]. In other studies, CD38 ligation by agonistic mAb in NK cells was also shown to induce calcium fluxes and tyrosine phosphorylation, as well as induce NK effector function including release of IFN- $\gamma$ and GM-CSF and cytotoxic responses leading to granzyme and cytokine release $[14,15]$. The cellular function of CD38 and its strong expression on MM cells has made CD38 an ideal therapeutic target for the treatment of MM.

\section{Daratumumab in preclinical studies}

Daratumumab is an immunoglobulin G1 kappa (IgG1k) human $\mathrm{mAb}$ that binds to a unique $\mathrm{CD} 38$ epitope on CD38-expressing cells with high affinity and was developed by the immunization of human immunoglobulin transgenic mice with recombinant CD38 protein [16]. de Weers et al. found that daratumumab was the only antibody in a panel of 42 human CD38-specific mAbs that triggered complement-dependent cytotoxicity (CDC) of Daudi target cells [16]. Thus, daratumumab was studied in a series of in vitro assays and was found to induce CDC in freshly isolated MM cells obtained from the bone marrow of 13 previously untreated or relapsed MM patients [16]. Furthermore, daratumumab triggered antibody-dependent cell-mediated cytotoxicity (ADCC) in CD38-expressing MM cell lines in peripheral blood mononuclear cells (PBMCs) enriched for NK cells, as well as in patient MM cells in the presence of both autologous and allogeneic effector cells [16]. Importantly, daratumumab did not induce ADCC in CD38-negative cells, confirming its specificity. Notably, daratumumab was effective at inducing both CDC and ADCC against $\mathrm{MM}$ cells in the presence of bone marrow stromal cells, suggesting that daratumumab is active in the bone marrow microenvironment [16]. In vivo, daratumumab exhibited high efficacy in interrupting tumor growth in mouse xenograft models [16].

In further studies investigating the mechanism of action of daratumumab, Nijhof et al. evaluated daratumumabinduced $\mathrm{CDC}$ or ADCC in vitro in bone marrow samples of $144 \mathrm{MM}$ patients [17]. Of note, no difference was found in daratumumab-induced CDC or ADCC between newly diagnosed, relapsed/refractory, or lenalidomideand bortezomib-refractory $\mathrm{MM}$ patients, suggesting that resistance to prior therapies might not affect the efficacy of daratumumab [17]. Furthermore, the investigators in this study noted a significant association between the level of CD38 expression and daratumumab-induced CDC and ADCC. Nijhof and colleagues found all-trans retinoic acid (ATRA) to upregulate CD38 expression, and pre-treatment with ATRA of patient-derived MM cells significantly increased susceptibility to daratumumabinduced CDC and ADCC in vitro [17]. Likewise, ATRA augmented daratumumab activity in a humanized MM mouse model [17].

In addition to $\mathrm{CDC}$ and $\mathrm{ADCC}$, daratumumab has also been shown to induce antibody-dependent cellular phagocytosis (ADCP). In a study by Overdijk and colleagues, daratumumab induced phagocytosis by human macrophages in MM cell lines in vitro observed by flow cytometry, as well as in in vivo subcutaneous and intravenous leukemic xenograft mouse models [18]. Moreover, daratumumab triggered macrophage-mediated phagocytosis ex vivo in patient-derived MM cell samples [18]. ADCP thus might be an important mechanism of action of daratumumab in the bone marrow microenvironment given that tumor-associated macrophages in the marrow have been shown to have Fc-dependent anti-tumor function [19]. Other mechanisms of daratumumab-induced cell death have been studied, finding that FcR-mediated crosslinking of daratumumab induces apoptosis of CD38expressing tumor cells in vitro [20]. The mechanisms of action of daratumumab are shown in Fig. 1.

Preclinical studies have also investigated daratumumab together with other anti-myeloma agents, finding that daratumumab activity is enhanced in combination with agents such as lenalidomide. Given that daratumumab induces ADCC by NK cells and lenalidomide promotes NK cell activity, van der Veer et al. investigated daratumumab combined with lenalidomide on ADCC against MM cells [21]. In this study, van der Veer and colleagues used PBMCs untreated or treated with lenalidomide as effector cells against derived MM cell lines or primary MM cells obtained from patients' bone marrow [21]. They found that pre-treatment of PBMCs with lenalidomide significantly enhanced daratumumab-induced ADCC against derived MM cell lines and primary MM cells [21]. Furthermore, the investigators treated whole bone marrow mononuclear cells of MM patients containing plasma cells with lenalidomide or daratumumab alone vs. in combination, finding that the combination of lenalidomide and daratumumab was synergistic, producing a $20 \%$ increased effect on tumor lysis [21]. In a separate study, the synergism between lenalidomide and daratumumab on ADCC against MM cell lines was found to occur largely via NK cell activation by lenalidomide rather than direct lenalidomide effect [22]. Further synergistic effect was demonstrated in an in vivo humanized mouse model engrafted with MM cells from a lenalidomide- and bortezomibrefractory patient [22]. In this model, treatment with lenalidomide alone resulted in tumor growth compared to treatment with daratumumab alone which suppressed tumor growth, while lenalidomide in combination with daratumumab resulted in tumor reduction [22]. In another study, the combination of IPH2102, an anti-KIR antibody, with daratumumab and lenalidomide enhanced daratumumab-induced ADCC [23]. 


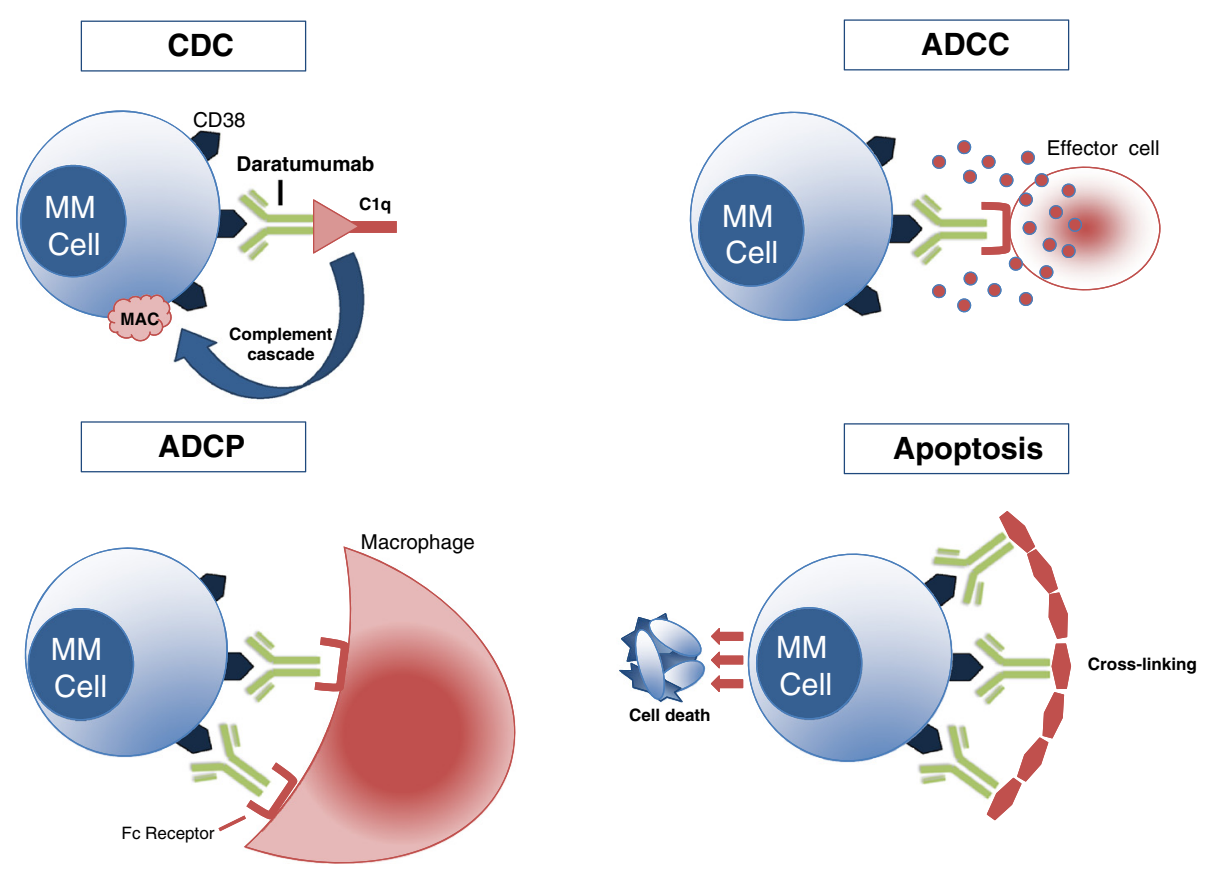

Fig. 1 Daratumumab mechanisms of action. Upper left: daratumumab binds CD38, and its Fc fragment is bound by C1q, initiating complement cascade and resulting in a MAC which leads to cell lysis and death. Upper right: daratumumab binds CD38, and its Fc fragment is then bound by an FcR-bearing effector cell, such as a natural killer cell, leading to activation of cytotoxic processes. Bottom left: daratumumab binds CD38, and its Fc fragment is then bound by an FcR-bearing macrophage, inducing phagocytosis. Bottom right: FcR-mediated crosslinking of daratumumab induces direct cellular apoptosis. MM cell multiple myeloma cell, CDC complement-dependent cytotoxicity, MAC membrane attack complex, ADCC antibody-dependent cell-mediated cytotoxicity, ADCP antibody-dependent cellular phagocytosis

\section{Daratumumab in clinical trials}

The promising anti-myeloma activity of daratumumab in preclinical studies led to the initiation of phase $1 / 2$ clinical trials with daratumumab both as monotherapy and in combination regimens. Clinical trials of daratumumab with available data are summarized in Table 1.

\section{Daratumumab as monotherapy}

The first-in-human clinical study of daratumumab (GEN501) was an open-label, multicenter, two-part, phase $1 / 2$ clinical trial of daratumumab as a single agent in patients with relapsed or relapsed and refractory myeloma evaluating its safety, efficacy, and pharmacokinetics [24]. Thirty two patients were enrolled in part 1 (doseescalation study), and 72 patients were enrolled in part 2 (dose-expansion study). In part 1 , the patients received daratumumab at doses of 0.005 to 24 milligrams per kilogram $(\mathrm{mg} / \mathrm{kg})$ in 10 cohorts; no maximum tolerated dose (MTD) was found. In the dose-expansion study, 72 patients were enrolled, and of these, 30 patients received daratumumab at a dose of $8 \mathrm{mg} / \mathrm{kg}$ and 42 patients received daratumumab at dose of $16 \mathrm{mg} / \mathrm{kg}$. The median age was 59 and 64 in the $8 \mathrm{mg} / \mathrm{kg}$ group and the $16 \mathrm{mg} / \mathrm{kg}$ group, respectively. The dose-expansion cohort was a heavily pre-treated population with a median of four prior therapies in both the 8 and $16 \mathrm{mg} / \mathrm{kg}$ dosing groups, and
$79 \%$ of patients were refractory to their last therapy. The overall response rate (ORR) was $36 \%$ in the $16 \mathrm{mg} / \mathrm{kg}$ group (with 2 complete responses [CRs], 2 very good partial responses [VGPRs], and 11 partial responses [PRs]), and the median duration of response (DOR) was not reached, with a reported 12-month progression-free survival (PFS) rate of $65 \%$ [22]. In the $8 \mathrm{mg} / \mathrm{kg}$ group, the ORR was only $10 \%$ (with three PRs) with a median DOR of 6.9 months [24]. The investigators performed a subgroup analysis of responses based on refractoriness to prior therapy in the $16 \mathrm{mg} / \mathrm{kg}$ group: $64 \%$ of the patients had disease refractory to both lenalidomide and bortezomib, and notably, the ORR among these doublerefractory patients was $33 \%$ [24]. The median PFS was 5.6 months in the $16 \mathrm{mg} / \mathrm{kg}$ group vs. 2.4 months in the $8 \mathrm{mg} / \mathrm{kg}$ group, and the 12 -month overall survival (OS) was $77 \%$ in both dosing groups [24]. In this heavily pre-treated and refractory patient population, daratumumab demonstrated encouraging anti-myeloma activity as a single agent as well as an acceptable safety profile. Infusion-related reactions (IRRs) occurred in $71 \%$ of patients, most during the first infusion. The majority of IRRs were grade 1 or 2 , with no treatment discontinuations due to IRR. Some of the most common grade 1 or 2 IRRs were pyrexia, influenza-like illness, fatigue, bronchospasm, nausea, and flushing. Overall, the most common non- 
Table 1 Clinical trials of daratumumab with available data

\begin{tabular}{|c|c|c|c|c|c|c|c|c|}
\hline Study & $\begin{array}{l}\text { NCT number } \\
\text { (trial name) }\end{array}$ & Phase & Number & Regimen & $\begin{array}{l}\text { ORR } \\
(\%)\end{array}$ & $\begin{array}{l}\text { PFS rate (\%) } \\
\text { (1 year) }\end{array}$ & $\begin{array}{l}\text { OS rate (\%) } \\
\text { (1 year) }\end{array}$ & $\begin{array}{l}\text { Median PFS } \\
\text { (months) }\end{array}$ \\
\hline \multicolumn{9}{|c|}{ Clinical trials with daratumumab as monotherapy } \\
\hline \multirow[t]{2}{*}{ Lokhorst et al. 2015 [22] } & \multirow[t]{2}{*}{ NCT00574288 (GEN501) } & \multirow[t]{2}{*}{$1 / 2$} & 30 & Daratumumab (8 mg/kg) & 10 & - & 77 & 2.4 \\
\hline & & & 42 & Daratumumab (16 mg/kg) & 36 & 65 & 77 & 5.6 \\
\hline Lonial et al. 2016 [23] & NCT01985126 (SIRIUS) & 2 & 106 & Daratumumab (16 mg/kg) & 29 & - & 65 & 3.7 \\
\hline \multicolumn{9}{|c|}{ Clinical trials with daratumumab in combination regimens } \\
\hline Plesner et al. 2015 [27] & NCT01615029 & $1 / 2$ & 32 & Daratumumab $(16 \mathrm{mg} / \mathrm{kg})+\mathrm{Rd}$ & 88 & - & - & - \\
\hline \multirow{4}{*}{$\begin{array}{l}\text { Chari et al. } 2015 \text { [29] } \\
\text { Mateos et al. } 2015 \text { [30] }\end{array}$} & \multirow[t]{4}{*}{ NCT01998971 } & \multirow[t]{4}{*}{1} & 6 & Daratumumab $(16 \mathrm{mg} / \mathrm{kg})+\mathrm{VD}$ & $100^{\mathrm{a}}$ & - & - & - \\
\hline & & & 11 & Daratumumab $(16 \mathrm{mg} / \mathrm{kg})+$ VTD & $100^{\mathrm{b}}$ & - & - & - \\
\hline & & & 8 & Daratumumab (16 mg/kg) + VMP & $100^{c}$ & - & - & - \\
\hline & & & 77 & Daratumumab $(16 \mathrm{mg} / \mathrm{kg})+$ POM-D & $59^{d}$ & - & - & - \\
\hline \multirow[t]{2}{*}{ Palumbo et al. 2016 [32] } & \multirow[t]{2}{*}{ NCT02136134 (CASTOR) } & \multirow[t]{2}{*}{3} & 251 & Daratumumab $(16 \mathrm{mg} / \mathrm{kg})+\mathrm{VD}$ & $83^{e}$ & - & - & $N R^{e}$ \\
\hline & & & 247 & VD & $63^{e}$ & - & - & $7.16^{\mathrm{e}}$ \\
\hline
\end{tabular}

ORR overall response rate, PFS progression-free survival, OS overall survival, $N R$ not reached, $R d$ lenalidomide and dexamethasone, VD bortezomib and dexamethasone, VTD bortezomib/thalidomide/dexamethasone, VMP bortezomib/melphalan/prednisone, POM-D pomalidomide/dexamethasone

${ }^{a}$ Median duration of follow-up days, 193

${ }^{\mathrm{b}}$ Median duration of follow-up days, 164

'Median duration of follow-up days, 267

${ }^{\mathrm{d}}$ ORR reported for 53 of 77 patients evaluable for efficacy; median duration of follow-up days, 148

e Median follow-up months, 7.4

hematologic adverse events (AEs) of all grades occurring in $\geq 25 \%$ of patients in both groups were fatigue, allergic rhinitis, pyrexia, diarrhea, upper respiratory tract infection, and dyspnea. The most common hematologic $\mathrm{AE}$ was neutropenia, occurring in $12 \%$ of patients in the $16 \mathrm{mg} / \mathrm{kg}$ cohort [24]. In pharmacokinetic analysis, $16 \mathrm{mg} / \mathrm{kg}$ was determined to be the lowest tested dose with consistent target saturation [24].

Daratumumab was further evaluated in an ongoing open-label, multicenter, phase II trial (SIRIUS) in patients with MM who had been treated with at least three prior lines of therapy or were refractory to both PIs and IMiDs [25]. In this study, patients were randomized to receive either daratumumab at 8 or $16 \mathrm{mg} / \mathrm{kg}$ in part 1 of the study. The $8 \mathrm{mg} / \mathrm{kg}$ cohort in part 1 did not meet criteria for dose expansion due to an ORR of only $11.1 \%$; thus, $16 \mathrm{mg} / \mathrm{kg}$ was the selected dose for further assessment in part 2 of the study [25]. In total, 106 patients received daratumumab $16 \mathrm{mg} / \mathrm{kg}$ in parts 1 and 2 . The cohort had a median age of 63.5 years and a median of five prior lines of therapy. Moreover, $95 \%$ of patients were refractory to the most recent PIs or IMiDs. The ORR was $29.2 \%$ (3 stringent complete responses [sCRs], 10 VGPRs, and 18 PRs) with a median DOR of 7.4 months [25]. Notably, ORR was $29.7 \%(30 / 101)$ in patients who were refractory to both PIs and IMiDs and $28.6 \%(20 / 70)$ in patients who were refractory to at least three agents (bortezomib, lenalidomide, carfilzomib, or pomalidomide) [25]. The median PFS was 3.7 months, and 12-month OS was $64.8 \%$ [25]. In this study, daratumumab demonstrated a favorable safety profile, with no treatment discontinuation due to drug-related AEs or IRR. The safety profile of both dosing cohorts was similar. In the $16 \mathrm{mg} / \mathrm{kg}$ group, the most common hematologic treatmentemergent AEs of any grade were anemia (33 \%), thrombocytopenia (25\%), neutropenia (23\%), and some of the most common non-hematologic treatment-emergent AEs were fatigue, nausea, and cough. Forty two percent of patients experienced IRR, the majority of which were grade 1 or 2 . The most common IRRs were nasal congestion, throat irritation, cough, dyspnea, chills, and vomiting.

In a combined analysis including 148 patients from the GEN501 and SIRIUS studies, the ORR was $31 \%$, and at a median follow-up of 14.8 months, the estimated OS was 19.9 months [26]. The GEN501 and SIRIUS trials demonstrated that daratumumab is active as monotherapy in patients with heavily pre-treated and relapsed/refractory myeloma (RRMM), with response rates comparing favorably to those of previous trials with pomalidomide or carfilzomib as monotherapy in RRMM [27, 28]. Importantly, daratumumab appears to maintain its anti-myeloma activity despite refractoriness to prior therapies, as seen by the encouraging ORR in patients who were refractory to both IMiDs and PIs in both studies (33 and $29.7 \%$ in GEN501 and SIRIUS, respectively) $[24,25]$. As a result of the SIRIUS trial, with findings in concordance with those of GEN501, the FDA approved daratumumab on November 16,2015 , for the treatment of refractory myeloma in patients who have received at least three previous lines of 
therapy, including a PI and an IMiD, or who are double refractory to a PI and IMiD.

\section{Daratumumab in combination regimens}

The enhanced anti-myeloma activity of daratumumab in combination with other agents in preclinical trials provided rationale for investigation of daratumumab in combination regimens.

Daratumumab in combination with lenalidomide and dexamethasone in patients with relapsed or relapsed and refractory myeloma is being studied in an ongoing, phase $1 / 2$, open-label, multicenter trial [29]. In part 1 , a $3+3$ design dose-escalation study, daratumumab was administered at doses of 2 to $16 \mathrm{mg} / \mathrm{kg}$ in combination with lenalidomide and dexamethasone until disease progression, unacceptable toxicity, or up to a maximum 24 months. Part 2 was a cohort expansion study, in which daratumumab was administered $16 \mathrm{mg} / \mathrm{kg}$ with lenalidomide and dexamethasone. Thirteen patients in total were enrolled in part 1, and MTD was not reached. Preliminary efficacy data from 20 patients presented at the 2014 American Society of Hematology (ASH) annual meeting showed marked decrease in $\mathrm{M}$ protein in all patients and a very encouraging ORR of $75 \%$ [30]. The last patient was enrolled in the study in August 2014, and updated efficacy analyses including a total of 32 patients enrolled in the part 2 expansion cohort have been presented [29]. The cohort had a median of two prior lines of therapy. The ORR was $88 \%$, with 11 PRs, 9 VGPRs, 1 $\mathrm{CR}$, and $7 \mathrm{sCRs}$ [29]. The median time to first response was 1 month and median time to best response was 4.5 months, indicative of deepening responses over time [29]. The median duration of follow-up was 7.8 months, and the median DOR was not reached, with $93 \%$ of responders remaining progression-free at time of analysis [29]. In this study, some of the most frequent reported treatment-emergent AEs were neutropenia, muscle spasms, cough, diarrhea, and fatigue. Grade 3 or 4 neutropenia was reported in $75 \%$ of patients. Fifty six percent of patients had mild to moderate IRR, which were most commonly cough, allergic rhinitis, nausea, vomiting, and dyspnea. Of two patients who had grade 3 IRR, one patient discontinued the study due to laryngeal edema.

Daratumumab is also under investigation in combination with other MM backbone regimens in an ongoing, open-label, four-arm, multicenter, phase 1b study $[31,32]$. The four arms of the study include daratumumab (at a dose of $16 \mathrm{mg} / \mathrm{kg}$ ) in combination with the following regimens: bortezomib/dexamethasone (VD), bortezomib/ thalidomide/dexamethasone (VTD), bortezomib/melphalan/prednisone (VMP), and pomalidomide/dexamethasone (POM-D). Inclusion criteria for each of the arms are as follows: VD and VTD arm (newly diagnosed MM, irrespective of transplant eligibility); VMP arm (newly diagnosed MM, transplant ineligible); and POM-D arm (relapsed/refractory to two or more lines of therapy including two or more consecutive cycles of lenalidomide and bortezomib) [32]. Preliminary efficacy results in newly diagnosed MM patients treated with daratumumab (DARA) in combination with VD $(n=6)$, VTD $(n=11)$, and VMP $(n=8)$ were reported by Mateos et al., with an ORR of $100 \%$ in each arm (DARA + VD: 3 PRs, 3 VGPRs; DARA + VTD arm: 7 PRs, 2 VGPRs, and 1 CR; DARA + VMP: 4 PRs, 4 VGPRs) [32]. Median duration of follow-up days was 193, 164, and 267 days for DARA + VD, DARA + VTD, and DARA + VMP, respectively [32]. Chari et al. reported an updated efficacy analysis of the DARA + POMD arm at the 2015 ASH annual meeting [31]. A total of 77 patients were enrolled (enrollment ongoing) at the time of the report in the POM-D arm, with median number of 3.5 prior therapies. Of the cohort, $65 \%$ were refractory to both a PI and IMiD. Of the 77 patients enrolled, 53 were evaluable for efficacy. The ORR was $58.5 \%$, with 3 sCRs, 1 CR, 12 VGPRs, and 15 PRs [31]. After a median follow-up of 148 days, 4/31 responders progressed [31]. Notably, the combination of daratumumab with POM-D exhibited remarkable efficacy among 40 evaluable double-refractory patients, with a $57.5 \%$ ORR [31]. In the POM-D arm, $61 \%$ of patients experienced IRR, which were most commonly chills, cough, and dyspnea. Otherwise, no significant additional toxicity was noted when DARA was added to POM-D. Some of the most common AEs of any grade were neutropenia, anemia, fatigue, cough, nausea, dyspnea, and diarrhea. Grade 3 or 4 AEs occurring in $>10 \%$ of patients were all hematologic toxicities (neutropenia [50.6\%], anemia [20.8\%], leukopenia [15.6\%], and thrombocytopenia [10.4\%]).

The remarkable efficacy of DARA in combination with both lenalidomide/dexamethasone and POM-D in RRMM and in combination with backbone regimens in newly diagnosed $\mathrm{MM}$ has provided rationale for initiation of phase III trials. MMY3003 (POLLUX) and MMY3004 (CASTOR) are currently ongoing randomized, open-label, multicenter, phase III trials for patients with relapsed or refractory MM, in which MMY3003 will compare the efficacy of daratumumab in combination with lenalidomide and dexamethasone vs. lenalidomide and dexamethasone alone, and MMY3004 will compare the efficacy of daratumumab in combination with bortezomib and dexamethasone vs. bortezomib and dexamethasone alone [33]. An interim analysis of the phase III CASTOR (MMY3004) trial presented at the 2016 American Society of Clinical Oncology annual meeting showed that daratumumab significantly improved ORR, PFS, and time to progression (TTP) in combination with bortezomib and dexamethasone [34]. A total of 498 patients with relapsed or refractory myeloma were randomized to receive daratumumab in combination with bortezomib and dexamethasone or 
bortezomib and dexamethasone alone. The ORR was $83 \%$ in patients who received daratumumab, bortezomib, and dexamethasone compared to $63 \%$ in patients who received bortezomib and dexamethasone alone $(p<0.0001)$ [34]. With a median follow-up of 7.4 months, median PFS and TTP in the daratumumab arm was not reached, whereas the median PFS and TTP in the bortezomib and dexamethasone arm was 7.16 and 7.29 months, respectively [34]. The combination of daratumumab, bortezomib, and dexamethasone significantly improved both PFS (HR 0.39; $95 \%$ CI, $0.28-0.53 ; p<0.0001$ ) and TTP (HR 0.30; 95 \% CI, 0.21-0.43; p < 0.001) compared to bortezomib and dexamethasone alone [34]. IRR occurred in $45 \%$ of patients, and the most common grade $3 / 4$ AEs were hematologic toxicities, with higher rates of thrombocytopenia (45 vs. $33 \%$ ) and neutropenia (13 vs. $4 \%$ ) occurring in the daratumumab arm.

The efficacy of daratumumab in newly diagnosed MM is also to be studied in a randomized, open-label, multicenter, phase III trial (Alcyone) that will compare VMP (bortezomib, melphalan, prednisone) vs. DARA + VMP in patients with newly diagnosed, previously untreated MM who are ineligible for high-dose therapy with stem-cell transplantation [35]. In another randomized, phase III trial (Cassiopeia), newly diagnosed transplant-eligible patients will be treated with VTD with or without daratumumab as induction therapy prior to transplant followed by VTD with or without daratumumab as consolidation therapy and then re-randomized to daratumumab maintenance therapy vs. observation. The ongoing clinical trials of daratumumab are listed in Table 2.

\section{Daratumumab interference with laboratory testing}

Daratumumab has been found to interfere with serum protein electrophoresis (SPEP) and immunofixation electrophoresis (IFE) assays due to co-migration of daratumumab with patients' $M$ protein, particularly with IgGk $M$ proteins, which impedes accurate quantification of endogenous $M$ protein [36]. This poses a clinical problem given SPEP and IFE assays are necessary for assessment of disease response in MM. A clinical assay, the daratumumab IFE reflex assay (DIRA), has been developed to mitigate daratumumab interference with IFE [36]. DIRA uses a mouse anti-daratumumab antibody that binds to daratumumab and shifts the migration of daratumumab away from the $M$ protein [36]. The specificity, reproducibility, and concordance of DIRA to distinguish daratumumab $\mathrm{mAb}$ from endogenous $\mathrm{M}$ protein were validated in a separate study [37]. The DIRA assay has been utilized in clinical trials of daratumumab to confirm disease response [24].

It has also been observed that the plasma of daratumumab-treated patients demonstrates positive antibody screens and panreactivity on blood compatibility testing as a result of daratumumab binding to CD38 on reagent red blood cells (RBCs) [38]. This finding is clinically relevant as MM patients undergoing treatment with daratumumab may, at some point, require blood

Table 2 Ongoing clinical trials of daratumumab in multiple myeloma

\begin{tabular}{|c|c|c|c|c|}
\hline NCT number & Title & Phase & Number & Recruitment \\
\hline NCT02116569 & A study of daratumumab in Japanese participants with relapsed or refractory multiple myeloma & 1 & 9 & Completed \\
\hline NCT02497378 & $\begin{array}{l}\text { A study of JNJ-54767414 (daratumumab) in combination with bortezomib and dexamethasone } \\
\text { (D-Vd) in Japanese participants with relapsed or refractory multiple myeloma }\end{array}$ & 1 & 6 & Recruiting \\
\hline NCT02519452 & $\begin{array}{l}\text { A study of daratumumab with the addition of recombinant human hyaluronidase (rHuPH20) } \\
\text { for the treatment of participants with relapsed or refractory multiple myeloma }\end{array}$ & 1 & 128 & Recruiting \\
\hline NCT02626481 & $\begin{array}{l}\text { Study of daratumumab in combination with dexamethasone in resistant or refractory } \\
\text { multiple myeloma }\end{array}$ & 2 & 64 & Recruiting \\
\hline NCT02316106 & $\begin{array}{l}\text { A study to evaluate } 3 \text { dose schedules of daratumumab in participants with smoldering } \\
\text { multiple myeloma }\end{array}$ & 2 & 120 & Recruiting \\
\hline NCT02419118 & $\begin{array}{l}\text { Monoclonal antibodies for treatment of multiple myeloma. Emphasis on the CD38 antibody } \\
\text { daratumumab }\end{array}$ & $2 / 3$ & 50 & Recruiting \\
\hline NCT02076009 & $\begin{array}{l}\text { A study comparing daratumumab, lenalidomide, and dexamethasone with lenalidomide and } \\
\text { dexamethasone in relapsed or refractory multiple myeloma }\end{array}$ & 3 & 571 & Active, not recruiting \\
\hline NCT02136134 & $\begin{array}{l}\text { Addition of daratumumab to combination of bortezomib and dexamethasone in participants } \\
\text { with relapsed or refractory multiple myeloma }\end{array}$ & 3 & 497 & Active, not recruiting \\
\hline NCT02541383 & $\begin{array}{l}\text { A study to evaluate daratumumab in transplant eligible participants with previously untreated } \\
\text { multiple myeloma (Cassiopeia) }\end{array}$ & 3 & 1080 & Recruiting \\
\hline NCT02252172 & $\begin{array}{l}\text { Study comparing daratumumab, lenalidomide, and dexamethasone with lenalidomide and } \\
\text { dexamethasone in participants with previously untreated multiple myeloma }\end{array}$ & 3 & 730 & Recruiting \\
\hline NCT02195479 & $\begin{array}{l}\text { A study of combination of daratumumab and Velcade (bortezomib) melphalan-prednisone } \\
\text { (DVMP) compared to Velcade melphalan-prednisone (VMP) in participants with previously } \\
\text { untreated multiple myeloma }\end{array}$ & 3 & 700 & Recruiting \\
\hline
\end{tabular}


transfusions and accurate detection of alloantibodies in blood compatibility testing is imperative in preventing blood transfusion reactions. Techniques by which to ameliorate daratumumab interference with blood compatibility testing are being investigated. Treating reagent RBCs with dithiothreitol (DTT), which denatures CD38, or the addition of neutralizing agents such as antiidiotype antibodies against daratumumab or recombinant soluble CD38 to patients' plasma has been found to inhibit blood compatibility testing interference [38, 39]. The results of a multicenter, international validation study on the use of DTT to resolve daratumumab interference with blood compatibility testing were presented at the 2015 ASH annual meeting [40]. In this study, participating blood banks received two unknown plasma samples, one sample with daratumumab and the other sample with daratumumab and an unknown clinically significant RBC antibody. The blood banks then performed standard antibody screening as well as antibody screening with DTT-treated RBCs. Of 23 participating sites, all sites reported daratumumab interference with standard antibody screening (gel, tube, or solid phase testing) but no interference using DTT-treated RBCs [40]. Using the DTT method, all 23 sites were able to correctly identify the unknown RBC antibody [40]. As DTT is known to also denature Kell antigens, study investigators recommend that $\mathrm{K}$ negative blood be administered when using the DTT method $[38,40]$. As the use of therapeutic antibodies for the treatment of $\mathrm{MM}$ increases, clinicians must remain mindful of potential laboratory interference with daratumumab as well as other mAbs.

\section{Conclusions}

Daratumumab has been well tolerated and has demonstrated encouraging response rates in clinical trials both as a single agent and in combination regimens in the relapsed/refractory setting $[24,25,29,31]$. Moreover, daratumumab in combination regimens has maintained a favorable safety profile without significant increase in toxicities [29, 31,34]. Clinical trials with other CD38-targeting mAbs isatuximab (or SAR650984) and MOR202 have also shown promising results [41-43]. Phase III trials of daratumumab both in the relapsed/refractory setting as well as in newly diagnosed patients will help to elucidate the role of daratumumab in the treatment of MM. Given the remarkable efficacy that has been seen with daratumumab in early clinical trials, daratumumab as well as other mAbs are likely to change the landscape of myeloma treatment.

\section{Abbreviations}

ADCC, antibody-dependent cell-mediated cytotoxicity; ADCP, antibodydependent cellular phagocytosis; AE, adverse event; ATRA, all-trans retinoic acid; CDC, complement-dependent cytotoxicity; CR, complete response; DARA, daratumumab; DIRA, daratumumab IFE reflex assay; DOR, duration of response; DTT, dithiothreitol; GM-CSF, granulocyte-macrophage colonystimulating factor; IFE, immunofixation electrophoresis; IMiD, immunomodulatory drug; IRRs, infusion-related reactions; mAbs, monoclonal antibodies; MM, multiple myeloma; MTD, maximum tolerated dose; NK, natural killer; ORR, overall response rate; OS, overall survival; PBMC, peripheral blood mononuclear cell; PFS, progression-free survival; PIs, proteasome inhibitors; POM-D, pomalidomide/dexamethasone; PR, partial response; $\mathrm{RBC}$, red blood cell; $S C R$, stringent complete response; SPEP, serum protein electrophoresis; VD, bortezomib/dexamethasone; VGPR, very good partial response; VMP, bortezomib/melphalan/prednisone; VTD, bortezomib/thalidomide/ dexamethasone

\section{Acknowledgements}

Yucai Wang is a recipient of Chinese American Hematologists and Oncologists (CAHON) Young Investigator Award (YIA). The authors would like to thank the Steve and Nancy Fox Research Fund for their generous support in lymphoma and myeloma research.

\section{Funding}

None.

\section{Availability of data and materials}

The dataset supporting the conclusions of this article is included within the article.

\section{Authors' contributions}

MW designed the study. LS and YW drafted the manuscript. LS designed and finalized the figure. DS and MW participated in the manuscript preparation and revisions. LS and YW contributed equally to this work. All authors read and approved the final manuscript.

\section{Competing interests}

The authors declare that they have no competing interests.

\section{Author details}

${ }^{1}$ Department of Medicine, Rutgers New Jersey Medical School, Newark, NJ, USA. ${ }^{2}$ Division of Multiple Myeloma, John Theurer Cancer Center, Hackensack University Medical Center, Hackensack, NJ, USA. ${ }^{3}$ Department of Lymphoma/ Myeloma, Division of Cancer Medicine, University of Texas M. D. Anderson Cancer Center, 1515 Holcombe Blvd., Unit 429, Houston, TX 77030, USA.

Received: 23 May 2016 Accepted: 24 June 2016

Published online: 30 June 2016

\section{References}

1. Kumar SK, Rajkumar SV, Dispenzieri A, Lacy MQ, Hayman SR, Buadi FK, et al. Improved survival in multiple myeloma and the impact of novel therapies. Blood. 2008;111(5):2516-20.

2. Kharfan-Dabaja MA, Hamadani M, Rejlic T, Nishihori T, Bensinger W, Djulbegovic B, et al. Comparative efficacy of tandem autologous versus autologous followed by allogeneic hematopoietic cell transplantation in patients with newly diagnosed multiple myeloma: a systematic review and meta-analysis of randomized controlled trials. J Hematol Oncol. 2013;6:2.

3. Kumar S, Lee JH, Lauerta JJ, Morgan G, Richardson PG, Crowley J, et al. Risk of progression and survival in multiple myeloma relapsing after therapy with IMiDs and bortezomib: a multicenter international myeloma working group study. Leukemia. 2012;26(1):149-57.

4. Kellner J, Liu B, Kang Y, Li Z. Fact or fiction-identifying the elusive multiple myeloma stem cell. J Hematol Oncol. 2013;6:91.

5. Sondergeld P, van de Donk NWCJ, Richardson PG, Plesner T. Monoclonal antibodies in myeloma. Clin Adv Hematol Oncol. 2015;13:599-609.

6. Malavasi F, Deaglio S, Funaro A, Ferrero E, Horenstein AL, Ortolan E, et al. Evolution and function of the ADP ribosyl cyclase/CD38 gene family in physiology and pathology. Physiol Rev. 2008;88:841-86.

7. Malavasi F, FunaroA RS, Horenstein A, Calosso L, Mehta K. Human CD38: a glycoprotein in search of a function. Immunol Today. 1994;15:95-7.

8. Lin P, Owens R, Tricot G, Wilson CS. Flow cytometric immunophenotypic analysis of 306 cases of multiple myeloma. Am J Clin Pathol. 2004;121:482-8.

9. Deaglio S, Mehta K, Malavasi F. Human CD38: a (r)evolutionary story of enzymes and receptors. Leuk Res. 2001;25:1-12. 
10. Dianzani U, Funaro A, DiFranco D, Garbarino G, Bragardo M, Redoglia V, et al. Interaction between endothelium and CD4 ${ }^{+}$CD45RA ${ }^{+}$lymphocytes Role of the human CD38 molecule. J Immunol. 1994;153:952-9.

11. Funaro A, De Monte LB, Dianzani U, Forni M, Malavasi F. Human CD38 is associated to distinct molecules which mediate transmembrane signaling in different lineages. Eur J Immunol. 1993;23:2407-11.

12. Deaglio S, Morra M, Mallone R, Ausiello CM, Prager E, Garbarino G, et al. Human CD38 (ADP-ribosyl cyclase) is a counter-receptor of CD31, an lg superfamily member. J Immunol. 1998;160:395-402.

13. Ausiello CM, la Sala A, Ramoni C, Urbani A, Funaro A, Malavasi F. Secretion of IFN- $\gamma$, IL-6, granulocyte-macrophage colony-stimulating factor and IL-10 cytokines after activation of human purified T lymphocytes upon CD38 ligation. Cell Immunol. 1996;173:192-7.

14. Mallone R, Funaro A, Zubiaur M, Baj G, Ausiello CM, Tacchetti C, et al. Signaling through CD38 induces NK cell activation. Int Immunol. 2001;13:397-409.

15. Sconocchia G, Titus JA, Mazzoni A, Visintin A, Pericle F, Hicks S, et al. CD38 triggers cytotoxic responses in activated human natural killer cells. Blood. 1999;94:3864-71.

16. de Weers $M$, Tai YT, van der Veer MS, Bakker JM, Vink T, Jacobs DCH, et al, Daratumumab, a novel therapeutic human CD38 monoclonal antibody, induces killing of multiple myeloma and other hematological tumors. J Immunol. 2011;186:1840-8.

17. Nijhof IS et al. Upregulation of CD38 expression on multiple myeloma cells by all-trans retinoic acid improves the efficacy of daratumumab. Leukemia. 2015;29:2039-49.

18. Overdijk MB, Verploegen $S$, Bögels $M$, van Egmond $M$, van Bueren JJ $L$, Mutis T, et al. Antibody-mediated phagocytosis contributes to the antitumor activity of the therapeutic antibody daratumumab in lymphoma and multiple myeloma. MAbs. 2015;7:311-20.

19. Grugan KD, McCabe FL, Kinder M, Greenplate AR, Harman BC, Ekert JE, van Rooijen N, Anderson GM, Nemeth JA, Strohl WR, et al. Tumor-associated macrophages promote invasion while retaining Fc-dependent anti-tumor function. J Immunol. 2012;189:5457-66.

20. Jansen JH, Boross P, Overdijk MB, van Bueren JJ, Parren PW, Leusen JH. Daratumumab, a human CD38 antibody induces apoptosis of myeloma tumor cells via Fc receptor-mediated crosslinking. Blood. 2012;120:2974

21. van der Veer $M$, de Weers $M$, van Kessel B, Bakker JM, Wittebol S, Parren PWHI, et al. Towards effective immunotherapy of myeloma: enhanced elimination of myeloma cells by combination of lenalidomide with the human CD38 monoclonal antibody daratumumab. Haematologica. 2011;96:284-90.

22. Nijhof IS, Groen RWJ, Noort WA, van Kessel B, de Jong-Korlaar R, Bakker A. Preclinical evidence for the therapeutic potential of CD38-targeted immune-chemotherapy in multiple myeloma patients refractory to lenalidomide and bortezomib. Clin Cancer Res. 2015;21:2802-10.

23. Nijhof IS, Lammerts van Bueren JJ, van Kessel B, Andre P, Morel Y, Lokhorst HM, et al. Daratumumab-mediated lysis of primary multiple myeloma cells is enhanced in combination with the human anti-KIR antibody IPH2102 and lenalidomide. Haematologica. 2015;100:263-8.

24. Lokhorst HM, Plesner T, Laubach JP, Nahi P, Gimsing M, Hansson MC, et al. Targeting CD38 with daratumumab monotherapy in multiple myeloma. N Engl J Med. 2015;373:1207-19.

25. Lonial S, Weiss BM, Usmani SZ, Singhal S, Chari A, Bahlis NJ, et al. Daratumumab monotherapy in patients with treatment-refractory multiple myeloma (SIRIUS): an open-label, randomized, phase 2 trial. Lancet. 2016

26. Usmani S, Weiss B, Bahlis NJ, Belch A, Lonial S, Lokhorst $\mathrm{H}$, et al. Clinical efficacy of daratumumab monotherapy in patients with heavily pretreated relapsed or refractory multiple myeloma. Blood. 2015;126(23):29.

27. Richardson PG, Siegel DS, Vij R, Hofmeister CC, Baz R, Jagannath S, et al. Pomalidomide alone or in combination with low-dose dexamethasone in relapsed and refractory multiple myeloma: a randomized phase 2 study. Blood. 2014;123:1826-32.

28. Siegel DS, Martin T, Wang M, Vij R, Jakubowiak AJ, Lonial S, et al. A phase 2 study of single-agent carfilzomib (PX-171-003-A1) in patients with relapsed and refractory multiple myeloma. Blood. 2012;120:2817-25.

29. Plesner T, Arkenau HT, Gimsing P, Krejcik J, Lemech C, Minnema MC, et al. Daratumumab in combination with lenalidomide and dexamethasone in patients with relapsed or relapsed and refractory multiple myeloma: updated results of a phase 1/2 study (GEN503). Blood. 2015;126(23):507.

30. Plesner T, Arkenau HT, Lokhorst HM, Gimsing P, Krejcik J, Lemech C, et al. Safety and efficacy of daratumumab with lenalidomide and dexamethasone in relapsed or relapsed, refractory multiple myeloma. Blood. 2014;124(21):84.
31. Chari A, Lonial S, Suvannasankha A, Fay JW, Arnulf B, Ifthikharuddin JJ, et al. Open-label, multicenter, phase $1 \mathrm{~b}$ study of daratumumab in combination with pomalidomide and dexamethasone in patients with at least 2 lines of prior therapy and relapsed or relapsed and refractory multiple myeloma. Blood. 2015;126(23):508.

32. Mateos MV, Moreau P, Comenzo R, Bladé J, Benboubker L, de la Rubia J, et al. An open-label, multicenter, phase $1 \mathrm{~b}$ study of daratumumab in combination with pomalidomide-dexamethasone and with backbone regimens in patients with multiple myeloma [abstract]. Haematologica. 2015;100(s1):84.

33. Palumbo A, Dimopoulos MA, Reece DE, Sonneveld P, Spencer A, ChananKhan AAA, et al. Twin randomized studies of daratumumab (DARA; D) plus standard of care (lenalidomide/dexamethasone or bortezomib/ dexamethasone [DRd or DVd]) versus $\mathrm{Rd}$ or $\mathrm{Vd}$ alone in relapsed or refractory multiple myeloma (MM): 547674MMY3003 (Pollux) and 54767414MMY3004 (Castor). J Clin Oncol. 2015:33:TPS8609.

34. Palumbo A, Chanan-Khan AAA, Weisel K, Nooka AK, Masszi T, Beksac M, et al. Phase III randomized control study of daratumumab, bortezomib, and dexamethasone (DVd) versus bortezomib and dexamethasone (Vd) in patients (pts) with relapsed or refractory multiple myeloma (RRMM): CASTOR study. J Clin Oncol. 2016;34:LBA4.

35. Mateos MV, Cavo M, Jakubowiak AJ, Carson RL, Qi M, Bandekar R, et al. A randomized open-label study of bortezomib, melphalan, and prednisone (VMP) versus daratumumab (DARA) plus VMP in patients with previously untreated multiple myeloma (MM) who are ineligible for high-dose therapy: 54767414MMY3007 (Alcyone). J Clin Oncol. 2015;33:TPS8608.

36. Axel AE, McCudden CR, Xie H, Hall BM, Sasser AK. Development of clinical assay to mitigate daratumumab, and IgG1-monoclonal antibody, interference with serum immunofixation (IFE) and clinical assessment of $\mathrm{M}$ protein response in multiple myeloma. Cancer Res. 2014;74(19 suppl):2563.

37. McCudden C, Axel A, Slaets D, Frans S, Bald J, Schecter JM, et al. Assessing clinical response in multiple myeloma (MM) patients treated with monoclonal antibodies (mAbs): validation of a daratumumab IFE reflex assay (DIRA) to distinguish malignant M-protein from therapeutic antibody. J Clin Oncol. 2015;33:8590

38. Chapuy CI, Nicholson RT, Aguad MD, Chapuy B, Laubach JP, Richardson PG, et al. Resolving the daratumumab interference with blood compatibility testing. Transfusion. 2015:55:1545-54

39. Oostendorp M, Lammerts van Bueren JJ, Doshi P, Khan I, Ahmadi T, Parren PWHI, et al. When blood transfusion medicine becomes complicated due to interference by monoclonal antibody therapy. Transfusion. 2015;55:1555-62.

40. Chapuy Cl, Aguad MD, Nicholson RT, AuBuchon JP, Cohn CS, Delaney M, et al. International validation of dithiotreitol (DTT)-based method to resolve the daratumumab interference with blood compatibility testing. Blood. 2015:126(23):3567.

41. Martin T, Strickland SA, Glenn M, Zheng W, Daskalakis N, Mikhael JR. SAR650984, a CD38 monoclonal antibody in patients with selected CD38+ hematological malignancies: data from a dose-escalation phase I study. Blood. 2013:122(21):284

42. Martin I, Hsu K, Strickland SA, Glenn M, Mikhael JR, Charpentier E. A phase I trial of SAR650984, a CD38 monoclonal antibody, in relapsed or refractory multiple myeloma. J Clin Oncol. 2014;32(5S):8532.

43. Raab M, Chatterjee M, Goldschmidt H, Agis H, Blau JW, Einsele H, et al. A phase I/lla study of the human anti-CD38 antibody MOR202 (MOR03087) in relapsed or refractory multiple myeloma [abstract]. Clin Lymphoma Myeloma Leuk. 2015;15 suppl 3:e80. Abstract BP-058. 